\title{
Study on "Pre-analytical Errors in a Clinical Biochemistry Laboratory": The Hidden Flaws in Total Testing
}

\author{
Sushma BJ*, Shrikant C \\ Department of Biochemistry, Chandulal Chandrakar Medical College and Hospital Kachandur, Durg, Chhattisgarh, India
}

\begin{abstract}
Aim of the study: The aim and objective of the present study was, to enumerate and evaluate different types of preanalytical errors in the clinical biochemistry laboratory and to compare the frequency of errors in the pre-analytical phase of testing before and after training the technical staff posted in the clinical biochemistry laboratory.

Materials and methods: A prospective Study was conducted at Dept. of Biochemistry, CIMS, Bilaspur. Chhattisgarh Institute of Medical Sciences, a tertiary care hospital cum medical college in Bilaspur for the period of 4 months from August 2016 to December 2016. During this period, different types of pre-analytical errors were monitored.

Results: Of the 19,411 samples received during the study period, 670 samples were found to be unsuitable for testing, accounting for $3.45 \%$ of the rejection. All these samples were rejected due to different types of pre-analytical errors that are due to wrong identification $(0.26 \%)$, missing samples $(0.05 \%)$, draw from IV site $(0.07 \%)$, inadequate samples $(1.02 \%)$, wrong timing of sample collection $(0.06 \%)$, hemolysed samples $(1.83 \%)$ and lipemic samples $(0.28 \%)$.

Conclusion: Of all the samples received in the clinical biochemistry laboratory, the overall percentage of rejection is $3.45 \%$. We also found that, there was reduction in the frequency of errors before and after training the staff.
\end{abstract}

Keywords: Pre-analytical errors; Hemolysis; Rejection; Frequency of errors; Clinical biochemistry laboratory

\section{INTRODUCTION}

Central Clinical Laboratory is the backbone to the hospital set up, as it contributes significantly in making the right diagnosis to the right patient at right time and hence the right treatment, which affects the duration of hospital stay, early treatment response and the well-being of the patient. Modern day medicine practice is purely evidence based which focuses on the valid laboratory reports for the effective and timely management of patients [1]. Advancement in the automation along with point of care testing, in the laboratory testing has occupied utmost position in the modern health care, which has led to the drastic improvements in the performance and speed up of laboratory reports. Despite of advanced automation considerable error rates are at clinical diagnostic labs [1].

Laboratory testing involves mainly three phases: 1) Pre-analytical phase 2) Analytical Phase and 3) Post-analytical phase. The preanalytical phase encompasses all the processes from the time of a laboratory request made by the physician until the specimen is analyzed in the lab (e.g. patient preparation, blood drawing, sample transportation, centrifugation, dilutions etc.). The analytical stage involves the analysis of the analytes using automation especially in clinical biochemistry laboratory and validation of the test results. The post-analytical stage refers to the interpretation of the results by laboratory consultants and reporting to the clinicians via printed reports [2].

Laboratory errors might occur at any of these three phases, depending upon their source and time of presentation respectively. The pre-and post-analytical phases of the process account for $93 \%$ of the errors [3].

Laboratory errors in healthcare are of concern when they lead to actual or potential adverse outcomes for patients. Given the complex nature of healthcare and the difficulty in assessing the effect of a specific laboratory error on patient management, the prevalence of proven patient harm, is difficult to assess. Obvious extreme errors in qualitative test results as we see in histopathology, blood transfusion, microbiology, virology, genetic testing are easiest to measure but assessing the effect of quantitative errors in clinical biochemistry and hematology results is much more difficult. Such difficulties mean that; present measurements probably significantly

Correspondence to: Sushma BJ, Assistant Professor, Dept of Biochemistry, Chandulal Chandrakar Medical College and Hospital Kachandur, Durg, Chhattisgarh, India, Tel: 8197247109; E-mail: sushmabj1983@gmail.com

Received: December 30, 2018; Accepted: January 12, 2019; Published: January 19, 2019

Citation: Sushma BJ, Shrikant C (2019) Study on "Pre-analytical Errors in a Clinical Biochemistry Laboratory": The Hidden Flaws in Total Testing. Biochem Anal Biochem 8:374. doi: 10.35248/2161-1009.19.8.374.

Copyright: (C) 2019 Sushma BJ, et al. This is an open-access article distributed under the terms of the Creative Commons Attribution License, which permits unrestricted use, distribution, and reproduction in any medium, provided the original author and source are credited. 
underestimate the size of the problem considering the high volume of quantitative testing performed in clinical laboratories. However, recent studies have shown that up to $70 \%$ of the errors are related to preanalytical phase of laboratory testing. The most common preanalytical errors include inappropriateness of test order, patient identification error, timing errors in sampling and preparation, hemolytic samples, lipemic samples, inappropriate transport and inappropriate sample collection tubes [4].

The purpose of our study was to evaluate different types of preanalytical errors in the clinical biochemistry laboratory and to compare the frequency of errors before and after training the technical staff posted in the clinical biochemistry laboratory.

\section{AIM AND OBJECTIVES OF THE STUDY}

The aim and objective of the present study was:

a. To enumerate and evaluate different types of pre-analytical errors in the clinical biochemistry laboratory.

b. To compare the frequency of errors in the pre-analytical phase of testing before and after training the technical staff posted in the clinical biochemistry laboratory.

\section{MATERIALS AND METHODS}

\section{Source of data}

A prospective Study On "Pre-Analytical Errors in a Clinical Biochemistry Laboratory" was conducted at Dept. of Biochemistry, CIMS, Bilaspur. Chhattisgarh Institute of Medical Sciences, a tertiary care hospital cum medical college in Bilaspur specializing in Medicine, Dermatology, Surgery, Pediatrics, Obstetrics and Gynecology, Psychiatry and Neurosurgery. The clinical biochemistry department is equipped with Automated Biochemistry Analyzer EM-360, Electrolyte Analyzers and Automated Immunoassay Analyzer (Beckman Coulter Access 2) and other ancillaries for sample processing and testing.

\section{Study protocol}

A total of 19,411 samples were analyzed in the clinical biochemistry laboratory for the period of 4 months from August 2016 to December 2016. All the technical staff and the ward nursing staff were trained in the month of October. Weekly 3 classes were held to educate the staff regarding sample collection, order of draw, pre-analytical variables and their influence on various parameters, Quality control checks and Quality control charts (LJ charts and westgard rules). A comparative study was done to know the frequency of pre-analytical errors before and after staff training.

During the study period, Individual sample was followed from the start of order of blood test to the final reporting of the test results. Each step of laboratory processing was recorded, which include patient preparation, test requisition form, specimen collection, specimen transportation, specimen preparation, specimen processing, software errors, instrument handling and maintenance, quality control check, reports interpretation, critical values and release of reports. All the lab personnel were instructed about the pre-analytical errors monitoring and documentation on daily basis. These errors were reviewed on weekly basis.

\section{Sample collection}

Inpatient blood samples were collected by the nursing staff in the respective wards and the outpatient blood samples were collected on site at centralized collection center by the lab personal. The samples were delivered to the lab by paramedical staff from wards and laboratory supportive staff from OPD respectively. OPD samples were collected by the lab personal, the samples were followed from the moment of sample draw to vacutainer transportation, centrifugation of vacutainers, separation of serum/ plasma and the analysis of sample. Sample collection procedure at OPD centralized collection center is as follows: Patients came along with the test requisition forms (TRF), after ensuring information on TRF, patients are asked to sit on regular chair for phlebotomy. Required amount of blood sample was collected using disposable syringes into the appropriate manually labelled (OPD number, name, age, gender) vacutainers. The sample collection timings were 10 AM-12 PM, all the filled vacutainers for Biochemistry tests were placed in a separate rack and were sent to Clinical Biochemistry Unit in the central laboratory for the sample processing and analysis. Once samples are reached to lab, they were centrifuged to obtain serum/plasma for test analysis. All the lab personal were instructed about the of monitoring and documentation preanalytical errors on daily basis.

Pre-analytical, Analytical and Post-analytical phases of testing were monitored. Analytical phase and post-analytical errors were monitored to ensure that these errors did not occur significantly in the present study. Analytical phase was also ensured with the calibrations and quality control runs (internal quality control and external quality controls). Fully Automated machines were used to analyze the samples. These equipments had inbuilt calibration traceability, these equipments were validated and verified, when they were installed. These equipments were maintained weekly or monthly as per the maintenance schedule provided by the vendor.

\section{Pre-analytical errors were monitored in this study are}

1. Incorrect sample identification: mismatch between the name on sample and test requisition form (TRF),

2. Missed Sample,

3. Sample from IV running area,

4. Inadequate Sample: sample received is not sufficient for testing,

5. Wrong timing for Collection - lipid profile,

6. Haemolysed Sample: Presence of pink to red tinge in serum or plasma,

7. Lipemic Sample: Presence of turbidity in serum or plasma caused by the accumulation of lipoprotein particles,

\section{Software errors.}

\section{Pre-assessment, training of technicians and post- assessment of technical staff}

All the study participants were given $10 \mathrm{MCQs}$ to assess the knowledge and skills. Time given for each question was 45 seconds. These MCQ questionnaire consisted of the questions which focused pre-analytical variables like Patient Preparation (fasting and post- prandial), Sample collection, Color coded tubes used for sample collection, Various anticoagulants used in various analysis, Dietary influence on certain parameters, Normal ranges of certain parameters. We had conducted several training classes weekly thrice various crucial topics like patient preparation, sample 
collection, order of draw, sample transportation, critical values, biomedical waste management, interfering factors in various biochemical assays, anticoagulants, arterial blood gas analysis, urine preservatives and syringe collection versus vacuum tube collection. After the training classes, again the knowledge and skills were assessed by questionnaire on these topics. Skills were assessed by Direct Observation: skills that were included under direct observation include; method of cleaning of the phlebotomy site by using $70 \%$ of Isopropyl alcohol in circular motion from inwards to outwards, usage of properly colour coded vacutainer tubes for various biochemical tests, method of blood draw, recapping of needles/destroying needle after usage. Training classes were conducted to all the technical staff in the month of October thrice a week, each session lasting for 2 hours duration. This training included hands on, series of lectures, MCQ discussions, project works and chart preparations. After, the training similar assessments were conducted as it was in pre-assessments.

\section{RESULTS}

A total of 19,411 samples were analyzed in the clinical biochemistry laboratory for the period of 4 months, which included both IPD and OPD samples, received from various clinical departments of our hospital. Out of 19,411 samples, pre-analytical errors were observed in 670 samples, which is approximately $3.45 \%$ of the total number of samples received. The distribution of different types of pre-analytical errors was calculated. Out of 19,411 samples, 51 samples were wrongly identified $(0.26 \%), 10$ samples were missing (0.05\%), 14 samples were drawn from IV running area $(0.07 \%)$, 198 samples were inadequate (1.02\%), 12 samples were collected in the wrong time $(0.06 \%), 356$ samples were hemolysed $(1.83 \%)$ and 55 samples were lipemic $(0.28 \%)$ as mentioned in Tables 1 and 2 .

Table 1: Laboratory total testing process and their potential errors.

\begin{tabular}{ll}
$\begin{array}{ll}\text { Testing phase } \\
\text { Pre-analytical } \\
\text { phase }\end{array}$ & Errors related to testing phase \\
& $\frac{\text { 1. Missed Test Requisition Form (TRF) }}{\text { 2. Incorrect sample identification }}$ \\
& $\frac{\text { 3. Incorrect sample tube }}{\text { 4. Sample from IV running area }}$ \\
& $\frac{\text { 5. Delay in sample transportation }}{\text { 6. Insufficient samples }}$ \\
& $\frac{\text { 7. Sample mix-ups }}{\text { 8. Tube broken in centrifuge }}$ \\
& $\begin{array}{l}\text { 9. Wrong timing for Collection } \\
\text { 10. Invalid Specimen: Haemolysed Sample, Lipemic } \\
\text { Sample and Icteric Sample }\end{array}$ \\
& 11. Software errors \\
\hline
\end{tabular}

Analytical phase 1 . Instrument not calibrated properly

2. Specimen mix-up

3. Inadequate specimen

4. Presence of interfering substances

5. Wrong analytical method

6. Lack of precision

Post-analytical 1. Wrong patient identification

phase

2. Report not legible

3. Report delayed

4. Transcriptional error

5. Specificity of the test not understood

6. Previous values are not available for comparison
All these samples were rejected for the testing and all these patients were advised for repeat fresh sample with proper TRF.

A total of 9970 samples were received for the analysis in 2 months from August to September, out of which 35 samples were wrongly identified $(0.35 \%), 6$ samples were missing $(0.06 \%), 9$ samples were drawn from IV running area $(0.09 \%), 168$ samples were inadequate $(1.68 \%), 8$ samples were collected in the wrong time (0.08\%), 228 samples were hemolysed $(2.28 \%)$ and 15 samples were lipemic $(0.15 \%)$ as mentioned in Table 3.

A training the staff in the month of October, a total of 9441 samples were received for the analysis in 2 months from November to December, out of which 16 samples were wrongly identified (0.17\%), 4 samples were missing $(0.04 \%), 5$ samples were drawn from IV running area $(0.05 \%), 30$ samples were inadequate $(0.37 \%), 4$ samples were collected in the wrong time $(0.04 \%), 128$ samples were hemolysed $(1.35 \%)$ and 40 samples were lipemic $(0.42 \%)$ as mentioned in Table 4.

Table 2: The distribution and frequency of pre-analytical errors observed in a total of 19,411 patients during 4 months.

\begin{tabular}{cccc}
\hline SL/No & $\begin{array}{c}\text { Type of pre-analytical } \\
\text { variable }\end{array}$ & Frequency & Percentage (\%) \\
\hline 1 & $\begin{array}{c}\text { Incorrect sample } \\
\text { identification }\end{array}$ & 51 & 0.26 \\
\hline 2 & Missed sample & 10 & 0.05 \\
\hline 3 & Sample from IV running area & 14 & 0.07 \\
\hline 4 & Inadequate sample & 198 & 1.02 \\
\hline 5 & Wrong timing of sample & 12 & 0.06 \\
\hline 6 & collection & 356 & 1.83 \\
\hline 7 & Haemolysed Sample & 55 & 0.28 \\
\hline
\end{tabular}

Table 3: The distribution and frequency of pre-analytical errors observed in a total of patients 9970 during 2 months (August and September).

\begin{tabular}{cccc}
\hline SL/No & $\begin{array}{c}\text { Type of pre-analytical } \\
\text { variable }\end{array}$ & Frequency & Percentage (\%) \\
\hline 1 & $\begin{array}{c}\text { Incorrect sample } \\
\text { identification }\end{array}$ & 35 & 0.35 \\
\hline 2 & Missed sample & 6 & 0.06 \\
\hline 3 & Sample from IV running area & 9 & 0.09 \\
\hline 4 & Inadequate sample & 168 & 1.68 \\
\hline 5 & Wrong timing of sample & 8 & 0.08 \\
\hline 6 & collection & 228 & 2.28 \\
\hline 7 & Haemolysed Sample & 15 & 0.15 \\
\hline
\end{tabular}

Table 4: The distribution and frequency of pre-analytical errors observed in a total of patients 9441 during 2 months (November and December).

\begin{tabular}{cccc}
\hline SL/No & $\begin{array}{c}\text { Type of pre-analytical } \\
\text { variable }\end{array}$ & Frequency & Percentage (\%) \\
\hline 1 & $\begin{array}{c}\text { Incorrect sample } \\
\text { identification }\end{array}$ & 16 & 0.17 \\
\hline 2 & Missed sample & 4 & 0.04 \\
\hline 3 & Sample from IV running area & 5 & 0.05 \\
\hline 4 & Inadequate sample & 30 & 0.37 \\
\hline 5 & Wrong timing of sample & 4 & 0.04 \\
\hline 6 & collection & 128 & 1.35 \\
\hline 7 & Haemolysed Sample & 40 & 0.42 \\
\hline
\end{tabular}


It is quite evident from Tables 3 and 4 that there was reduction in the frequency of errors before and after training the staff, with respect to Incorrect sample identification from $0.35 \%$ to $0.17 \%$, missed samples from $0.06 \%$ to $0.04 \%$, Sample from IV running area from $0.09 \%$ to $0.05 \%$, Inadequate sample from $1.68 \%$ to $0.37 \%$, Wrong timing of sample collection from $0.08 \%$ to $0.04 \%$ and Haemolysed Sample from $2.28 \%$ to $1.35 \%$. In case of lipemic samples it varied from $0.15 \%$ to $0.42 \%$.

\section{DISCUSSION}

Error rates are often described using the sigma concept, which refers to the number of standard deviations that lie between the process mean and the specification limit. Sigma $(\sigma)$ is a Greek alphabet letter, used to describe variability in a process. In the sixsigma methodology, the unit used is defects per unit. A sigma value indicates the frequency of defects occurring in a process. Therefore, a higher sigma value translates in lower defects and a lower sigma value means a higher number of defects. A process is cited to be performing at 'world class' levels when it is functioning at levels of six sigma [5]. In other words, a process performing at six sigma level translates into a phenomenal 3.4 Defects per Million (DPM) opportunities, the practical limit to perfection [6].

The analytical phase of laboratory medicine is arguably the best performing sector in healthcare with close to 5 sigma performance $(0.002 \%)$ [7]. This is more than 3,000 times lower than the rates of infection and medication errors and reflects the standardized quantitative nature of much of laboratory medicine testing, which is well suited to statistical quality control measures [8]. However, the accomplishments of laboratory medicine drop, when errors in all phases of the total testing process are considered $[9,10]$. The proportion of errors associated with the two extra-analytical phases is 4-5 times that seen in the analytical phase, with the pre-analytical phase consistently representing over half of all errors in published studies [11-15].

Advances in science and technology have led to many pathbreaking advances in the field of medical diagnostics, that have transformed laborious, manual and cumbersome testing methods into fully automated tests, which yields reliable, rapid, accurate and précised results. However, despite advances in analytical phase and post-analytical phase of testing (Laboratory Information System), still notable errors are happening particularly in the pre-analytical phase, due to which the results are affected, no matter how best is the performance of the automation. If the pre-analytical errors are not eliminated in the system, report reliability will be questionable.

Pre-analytical phase errors have been the focus of research in past decades. Previous studies have focused on the analytical phase of diagnostic tests, and many quality control programmes were initiated at diagnostic labs to monitor analytical phase errors. However post-and pre-analytical errors were neglected worldwide, and currently many studies are focusing on the importance of pre-analytical phase to obtain accurate lab results. An American Pathologist program conducted a study enrolling 660 laboratories and showed that order error rate from outpatient's center was $4.8 \%$ [16]. The College of American Pathologists, including 120 labs, concluded that misidentification is a common laboratory error. Another study conducted in the past by Danish on laboratory errors showed that $81 \%$ of the lab errors were pre-analytical, while only $10 \%$ of lab errors were analytical. Moreover, $82.6 \%$ human errors and $4.3 \%$ technical errors observed [17].
In our study, we observed $3.45 \%$ of different types and frequencies of pre-analytical errors in clinical biochemistry laboratory at our institute. Among all the types of pre-analytical errors, the most common error was found to be hemolysis, which accounted for $1.83 \%$ of total sample rejection which is similar to many other studies [18]. Hemolysis has a profound influence on various parameters like Potassium, Acid Phosphatase (ACP), Lactate Dehydrogenase (LDH), Aspartate Transaminase (AST), Alanine Transaminase (ALT), Creatinine, Creatine Kinase (CK), albumin, alkaline phosphatase (ALP), chloride, gamma-glutamyl transferase (GGT), glucose and sodium. Parameters like Potassium, Alanine Transaminase (ALT), Creatinine, Creatine Kinase (CK) are overestimated when hemolysed sample are used for analysis, whereas parameters like albumin, alkaline phosphatase (ALP), chloride, gamma-glutamyl transferase (GGT), glucose, bilirubin and sodium are underestimated when hemolysed sample are used for analysis. The various causes for hemolysis found to be Cleansing the venipuncture site with alcohol and not allowing the site to dry appropriately (at least $30 \mathrm{sec}$ ), syringe draws, vigorous mixing of the samples, transferring the sample into a tube by pushing down on the syringe plunger to force blood into a tube and not allowing the serum specimen to clot for the recommended amount of time can result in fibrin formation in the serum [19].

The next common error that we come across in our study was inadequate sample, accounting for $1.02 \%$ sample rejection. Every analytical process requires specified amount of serum/plasma for analysis. The main reason behind this error was the phlebotomist were lacking the knowledge about the testing volume, difficulty in sampling in pediatric cases, debilitating diseases, not reading the test requisition form properly by the laboratory personnel (number of tests requested in test requisition form), large number of patient's samples need to be collection in the specified timings and shortage of manpower.

Lipemic samples accounted for $0.15 \%$ of rejection. Lipemic samples arise due to wrong timing of sample collection (postmeals) and if a patient is diagnosed have hyperlipoproteimias. This can be avoided by advising for overnight fasting along with post one-week treatment overnight sample if it was not emergency and for emergency samples sample dilution method and interference testing assay was performed. In case of patient diagnosed to have hyperlipoproteinemia's, we requested the clinicians mention it on TRF. The direction and magnitude of lipemia interference in spectrophotometric assays depends on wavelength of the reaction and blanking of the method. Specially like AST, ALT, glucose which uses NADPH at $340 \mathrm{~nm}$ are affected. Lipemia results in falsely decreased levels of serum electrolytes due to high proportion of lipid in plasma (25\%) which is normally around $8 \%$ in plasma, hence water portion is only about $75 \%$ compared to $92 \%$ water in normal plasma [20].

Patient identification is the critical first step in blood collection. In the 2007 Laboratory Services, National Patient Safety Goals from The Joint Commission, goal \# 1 is accuracy in patient identification. Patient misidentification errors are potentially associated with the worst clinical outcomes because of the possibility of misdiagnosis and mishandled therapy. In our study, we found $0.26 \%$ Incorrectly identified samples which accounted for of the rejection. this may be probably, due to heavy work load and It is important to identify a patient accurately so that blood is collected from the correct person. Drawing blood from the wrong person, or labeling the correct patient's sample with a 
different patient's label can certainly contribute to laboratory error. When identifying the patient, have them provide their full name, address, identification number and/or date of birth. Hospital inpatients should be wearing an identification band with the above information, which the phlebotomist should confirm before the venipuncture. Phlebotomists should pleasantly introduce themselves to the patient and clearly explain the procedure to be performed. It is always a courtesy to speak a few words in a patient's native language if English is not their first language. It is necessary to have the patient state and speak his or her name. If a patient cannot provide this information, he or she must provide some form of identification or be identified by a family member or caregiver. Check the identification band that is physically attached to the patient. Wristbands with unique barcoded patient identifiers will have a great potential for reducing patient misidentification.

The other errors which accounted for rejection are, missing samples $(0.05 \%)$, samples drawn from IV area $0.07 \%$ and wrong timing of sample collection $0.08 \%$.

All the technical staff and the ward nursing staff were trained in the month of October. Weekly 3 classes were held to educate the staff regarding sample collection, order of draw, pre-analytical variables and their influence on various parameters, Quality control checks and Quality control charts (LJ charts and westgard rules). A comparative study was done to know the frequency of preanalytical errors before and after staff training. We found that after training the staff, there was reduction in the frequency of errors before and after training the staff, with respect to Incorrect sample identification from $0.35 \%$ to $0.17 \%$, missed samples from $0.06 \%$ to $0.04 \%$, Sample from IV running area from $0.09 \%$ to $0.05 \%$, Inadequate sample from $1.68 \%$ to $0.37 \%$, Wrong timing of sample collection from $0.08 \%$ to $0.04 \%$ and Haemolysed Sample from $2.28 \%$ to $1.35 \%$.

Icterus or hyperbilirubinemia is the presence of elevated bilirubin levels, occurs due to increased bilirubin production or inappropriate excretion as we see in hemolytic anemia, liver diseases or biliary tract obstruction. Icteric serum or plasma ranges in color from dark yellow to bright yellow, rather than normal straw color. The abnormal colour of the serum can interfere with photometric measurements because of its ability to react chemicals in other reagents resulting in decreased analyte values or spectral interferences during color measurement. Concentrations of bilirubin greater than $2.5 \mathrm{mg} / \mathrm{dL}$ can lead to clinically relevant changes of anti-thrombin. Higher concentrations can interfere with coagulation tests. In our study, were found $1.37 \%$ of icteric samples.

\section{CONCLUSION}

The concept of total testing process in the laboratory encompasses all the steps involved in the sample collection, sample processing, sample analysis, from the beginning of physician ordering the test to the final interpretation of the results. These days most of the studies focus on the minimization of pre-analytical errors, since these errors contribute to maximum in the testing process. Our study also recommends the training of phlebotomy staff on various aspects of sample collection, handling, processing, transportation and quality control. The promotion of ideal phlebotomy practices and sample transport procedures is a pre-requisite for the efficiency of laboratory functioning. The dependency on the accurate laboratory reports for diagnosing the disease makes it mandatory for labs to ensure accountability and accuracy of the reports to exclude the patients from incorrect diagnosis and incorrect treatment.

To conclude, we would like to state that all the laboratories running across the state or country should follow standard norms, run internal quality controls on daily basis and participate in external quality control program. All the staff working in the laboratory should be given appropriate training and participate in many continuing medical education programmes. Thus, government should make extra effort to ensure equal quality at public hospitals.

\section{REFERENCES}

1. Baron JM, Mermel CH, Lewandrwski KB, Dighe AS. Detection of preanalytical laboratory testing errors using statistically guided protocol. Am J Clin Pathol. 2012;138:406-413.

2. Cornes MP, Atherton J, Pourmaharam G, Borthwick H, Kyle B, West $\mathrm{J}$, et al. Monitoring and reporting of pre-analytical errors in laboratory medicine: the UK situation. Ann Clin Biochem. 2016;53:279-284.

3. Boone DJ. Governmental perspectives on evaluating laboratory performance. Clin Chem. 1933;39:1461-1467.

4. Sakyi A, Laing E, Ephraim R, Asibey O, Sadique O. Evaluation of analytical errors in a clinical chemistry laboratory: a 3-year experience. Ann Med Health Sci Res. 2015;5:8-12.

5. Harry MJ. "Six Sigma: A Breakthrough Strategy for Profitability." Quality Progress. 1998;31:60-64.

6. Eichhorn JH. Prevention of intraoperative anesthesia accidents and related severe injury through safety monitoring. Anesthesiology. 1989;70:572-577.

7. Leape LL. Errors in medicine. Clin Chim Acta. 2009;404:2-5.

8. Nevalainen D, Berte L, Kraft C, Leigh E, Picaso L, Morgan T, et al. Evaluating laboratory performance on quality indicators with the six sigma scale. Arch Pathol Lab Med. 2000;124:516-519.

9. Plebani M. Exploring the iceberg of errors in laboratory medicine. Clin Chim Acta. 2009;404:16-23.

10. Kalra J. Medical errors: impact on clinical laboratories and other critical areas. Clin Biochem. 2004;37:1052-1062.

11. Astion ML, Shojania KG, Hamill TR, Kim S, Ng VL. Classifying laboratory incident reports to identify problems that jeopardize patient safety. Am J Clin Pathol. 2003;120:18-26.

12. Kalra J. Medical errors: overcoming the challenges. Clin Biochem. 2004;37:1063-1071.

13. Rattan A, Lippi G. Frequency and type of pre-analytical errors in a laboratory medicine department in India. Clin Chem Lab Med. 2008;46:1657-1659.

14. Bonini P, Plebani M, Ceriotti F, Rubboli F. Errors in laboratory medicine. Clin Chem. 2002;48:691-698.

15. Szecsi PB, Odum L. Error tracking in clinical biochemistry laboratory. Clin Chem Lab Med. Walter de Gruyter. 2009;47:1253-1257.

16. Valenstein P, Meler F. Outpateint order accuracy. A College of American Pathologists Q-probes study of requisition order entry accuracy in 660 institutions. Arch Pathol Lab Med. 1999;123:1145. 1150.

17. Lippi G, Salvango GL, Montaganna M, Brocco G, Guidi GC. Influence of hemolysis on routine clinical chemical testing. Clin Chem Lab Med. 2006;44:311-316.

18. Koseoglu M, Hur A, Atay A, Cuhadar S. Effects of hemolysis interference on routine biochemistry parameters. Biochem Med. 2011;21:79-85. 
19. Calmarza P, Cordero J. Lipemia Interferences in routine clinical biochemical tests. Biochem Med. 2011;21:160-166.
20. Carraro P, Plebani M. Errors in a state laboratory: types and frequencies 10 years later. Clin Chem. 2007;53:1338-1342. 